\title{
Evolutive and structural characterization of Nostoc commune iron-superoxide dismutase that is fit for modification
}

\author{
Y. Ma, M. Lu, J.-Y. Li, Y. Qin and X.-G. Gong \\ Institution of Biochemistry, College of Life Sciences, Zhejiang University, \\ Hangzhou, P.R. China \\ Corresponding author: X.-G. Gong \\ E-mail: gongxingguo@126.com
}

Genet. Mol. Res. 11 (4): 3607-3617 (2012)

Received January 19, 2012

Accepted June 21, 2012

Published October 4, 2012

DOI http://dx.doi.org/10.4238/2012.October.4.8

\begin{abstract}
Superoxide dismutase (SOD) has extensive clinical applications for protecting organisms from toxic oxidation. In this study, the integrated iron-superoxide dismutase gene ( $f e-s o d)$ coding sequence of Nostoc commune stain CHEN was cloned from genomic DNA and compared to sods from other reported algae. These analyses of immunology and phylogenetics indicated that this Fe-SOD is considerably homologous with SODs from lower prokaryotes (FeSOD or Mn-SOD) but not those from higher animals (Cu/Zn-SOD). In addition, the $N$. commune $\mathrm{Fe}-\mathrm{SOD}$ shows 67 to $93 \%$ protein sequence identity to 10 other algal Fe-SODs (or Mn-SODs) and 69 to $93 \%$ gene sequence identity. Rare nonsynonymous substitutions imply that algal SODs are being subjected to strong natural selection. Interestingly, the $N$. commune Fe-SOD enzyme molecule has a compact active center that is highly conserved $(38.1 \%$ of residues are absolutely conserved), and 2 loose ends localized outside the molecule and inclined to mutate (only $11.5 \%$ of residues are absolutely conserved). Based on associative analyses of evolution, structure, and function, this special phenomenon is attributed to function-
\end{abstract}


dependent evolution through negative natural selection. Under strong natural selection, although the mutation is random on the gene level, the exterior region is inclined to mutate on the protein level owing to more nonsynonymous substitutions in the exterior region, which demonstrates the theoretical feasibility of modifying Fe-SOD on its ends to overcome its disadvantages in clinical applications.

Key words: Nostoc commune; Iron-superoxide dismutase; Phylogenetics; Structure and function; Natural selection

\section{INTRODUCTION}

The superoxide dismutase (SOD) family is a group of functionally anti-oxidative stress proteins found in all 3 kingdoms of life. Three classes of SODs occur (Fe-SOD, Mn-SOD, and Cu/ $\mathrm{Zn}-\mathrm{SOD}$ ), each differing in the metals at their catalytic active sites. Fe-SOD and Mn-SOD are found in Eubacteria, Eubacteria-derived eukaryotic mitochondria, and Archaebacteria (Regelsberger et al., 2004; Gottlieb et al., 2005; Brioukhanov et al., 2006; Chen et al., 2007; Seo et al., 2007). The $\mathrm{Cu} / \mathrm{Zn}$ enzyme is confined almost exclusively to the cytoplasm of eukaryotic organisms.

The theory of free radicals and tumors holds that reactive oxygen species originating in redundant radicals attack organisms, leading to genetic mutations and tumors (Behrend et al., 2003; Li et al., 2008; Takenaka et al., 2011). SOD is supposed to be the main cellular superoxide anion-degrading enzyme that inhibits tumor growth (Zhang et al., 2002; Hu et al., 2005). However, SOD has many critical disadvantages in clinical applications: it cannot target tumor cells (Oyanagui et al., 1991), resulting in a low curative efficacy, and it can barely permeate cell membranes because of its large molecular weight (Delanian et al., 2001; Jubeh et al., 2005; Rengel et al., 2005), which leads to a low cellular effect. In previous SOD modifications, we fused fe-sod with the LC-1 single-chain variable fragment antibody ( $\mathrm{ScFv}$ ) gene and achieved an SOD-ScFv fusion protein that could actively recognize SPC-A-1 (a human lung adenocarcinoma cell line) lung tumor cells and permeate cell membranes (Lu et al., 2006). However, the modification of SOD for better effectiveness is still worthy of further research and development.

As promising green foods in the 21st century, Nostoc commune and Spirulina platensis have abundant Fe-SOD enzymes (Shirkey et al., 2000; Choudhary et al., 2007) that we have purified and characterized in previous studies. SOD activity assays have indicated that the specific activities of these enzymes are 2700 and $4200 \mathrm{U} / \mathrm{mg}$, respectively. They have been applied to treat inflammation and cancer (Lu et al., 2006). Detailed investigation of the enzyme sequences and structures, which are related with catalytic function, is necessary to prepare them for further modification and application. In this study, we cloned fe-sod from $N$. commune stain CHEN and raised an anti-SOD antibody to conduct immunological and phylogenic analyses of Fe-SOD. To extend this analysis, we characterized the phylogenesis of additional genes of the SOD family from 14 lower prokaryotes and 4 representative eukaryotes. The evolution/structure/function-based predictive analysis in the current study presented an interesting and special evolution characterization, which we considered to be the result of a "special law of nature" under strong selection stress. This insight theoretically prepares us for modifications of SOD that overcome its current disadvantages. 


\section{MATERIAL AND METHODS}

The pUCm-T vector Escherichia coli BL21 (DE3): hsdS gal ( $\lambda$ cIts857 ind 1 Sam7 nin5 lacUV5-T7) was available at our institution. N. commune stain CHEN was cultivated in BG11 culture medium. E. coli BL21 colonies were maintained in Luria-Bertani culture medium. Cu/Zn-SOD (8000 U/mg) was obtained from Baoan Corporation in Shanghai, China.

\section{Cloning of $f e$-sod}

N. commune stain CHEN were grown in BG11 culture medium (150 mg NaNO, $4 \mathrm{mg}$ $\mathrm{K}_{2} \mathrm{HPO}_{4}, 7.5 \mathrm{mg} \mathrm{MgSO} \mathrm{Mg}_{4} .7 \mathrm{H}_{2} \mathrm{O}, 3.6 \mathrm{mg} \mathrm{CaCl} .2 \mathrm{H}_{2} \mathrm{O}, 5.8 \mathrm{mg} \mathrm{Na}_{2} \mathrm{SiO}_{3} .9 \mathrm{H}_{2} \mathrm{O}, 0.6 \mathrm{mg}$ citric acid, $0.6 \mathrm{mg}$ ferric ammonium citrate, $0.1 \mathrm{mg}$ ethylenediaminetetraacetic acid, $2 \mathrm{mg} \mathrm{Na} \mathrm{CO}_{3}, 0.1$ $\mathrm{mL} \mathrm{A}_{5}$ solution, and $100 \mathrm{~mL} \mathrm{H}_{2} \mathrm{O}$ ) at $25^{\circ} \mathrm{C}$ and exposed to light (intensity of $2000 \mathrm{Lux}$ ) each day for $10 \mathrm{~h}$. After 45 days, a $0.1 \mathrm{~g} N$. commune sample was taken, and its genomic DNA was isolated using the method of Kim et al. (1997). The SOD fragment was amplified from the genomic DNA and primed with SOD-forward (5'-GAGGATTTGACACGATGGCATTTG$3^{\prime}$ ) and SOD-backward (5'-CTGACACCTAATTAAGCTTTGGCC-3') according to a method published previously (Lu et al., 2006). The achieved sod was sequenced. Homology searches were performed with the Basic Local Alignment Search Tool (http://www.ncbi.nlm.nih.gov/ BLAST), and $13 \mathrm{Fe}-\mathrm{SODs}$ (or Mn-SODs) showing the best homology (including 10 reported SODs from algae) were recorded (see Table 1). These sequences were aligned using Clustal $\mathrm{X}$, and neighbor-joining (NJ) was performed using the Molecular Evolutionary Genetics Analysis 4 software to construct phylogenetic trees. Branch supports were estimated with a bootstrap analysis of 3000 replicates for NJ. Sequence alignments and analysis were carried out with the GeneDoc software. The tertiary structure was predicted by submitting the protein sequence to the SWISS-MODEL server (http://www.expasy.org/swissmod/SWISS-MODEL. html) through ExPASy, and the resultant tertiary model was visualized using RasMol 2.6.

\section{Immunoblot analyses of Fe-SOD from $N$. commune}

The native Fe-SOD protein was purified from N. commune stain CHEN (or S. platensis) using DEAE-Sepharose Fast Flow and Sephadex G-100 in an orderly manner (Lu et al., 2006). The SOD activity was assayed using the pyrogallol autoxidation method (Marklund and Marklund, 1974). The purified $N$. commune Fe-SOD was used to raise anti-SOD antiserum. The antiserum was then applied to react with SODs from N. commune, S. platensis, and cattle erythrocyte cells via Western blotting.

\section{RESULTS}

\section{Cloning and immunologic characterization of Fe-SOD from $N$. commune stain CHEN}

Primers were designed against the sequence ends of $f e$-sods from other algae (Nostoc linckia AAL25194 and Anabaena variabilis ATCC 29413, ABA20587). The achieved fe-sod sequence (accession No. AAV84021) is $603 \mathrm{bp}$ and contains initiation (ATG) and stop (TAA) 
codons. Purified native N. commune stain CHEN Fe-SOD was used to raise the anti-SOD antiserum. To ensure the activity of this antiserum, we reacted it with $N$. commune stain CHEN Fe-SOD. The result showed that this antiserum could effectively recognize $N$. commune stain CHEN Fe-SOD (Figure 1, lane 1). Moreover, the antiserum could recognize native Fe-SOD from $S$. platensis. However, it barely reacted with $\mathrm{Cu} / \mathrm{Zn}-\mathrm{SOD}$ from cattle erythrocyte cells. We, therefore, concluded that the Fe-SOD from $N$. commune stain CHEN was more antigenically related to that of $S$. platensis than to that of $\mathrm{Cu} / \mathrm{Zn}-\mathrm{SOD}$ from higher animals.

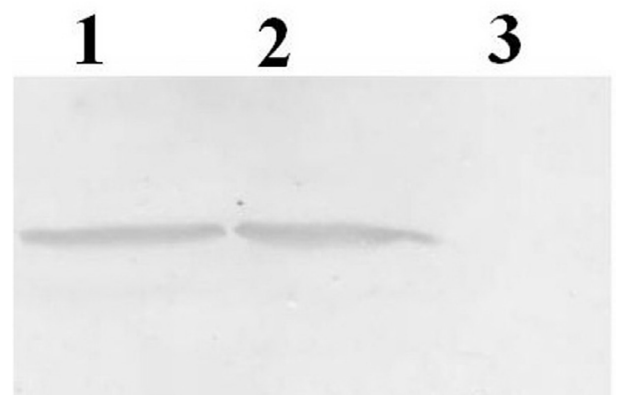

Figure 1. Immunology analysis of Nostoc commune Fe-SOD. Anti-SOD antiserum was raised as follows: $10 \mu \mathrm{g}$ purified $N$. commune Fe-SOD was used to immunize 10 male mice ( $20 \mathrm{~g} / \mathrm{animal})$. After 15 days, the mice were injected with a booster dose of additional $15 \mu \mathrm{g}$ protein. In the 30th day, they were bled from periorbital artery and the antiserum was extracted. Samples (lane $1=50 \mu \mathrm{g}$ Fe-SOD from $N$. commune strain CHEN; lane $2=50$ $\mu \mathrm{g}$ native Fe-SOD from Spirulina platensis; lane $3=50 \mu \mathrm{g}$ native Fe-SOD from cattle erythrocyte cells) were subjected to $10 \%(\mathrm{w} / \mathrm{v})$ SDS-PAGE and transferred to nitrocellulose membranes, and incubated with the anti-SOD antiserum (1:2000). Membranes were then incubated with secondary antibody (horseradish peroxide-conjugated goat anti-mouse antibody, 1:20,000), and visualized.

\section{Phylogenetic analysis}

We analyzed the phylogenetic relationships among 14 Fe-SODs (or Mn-SODs) from lower prokaryotes (including 10 reported SODs from algae) and 4 representative Cu/Zn-SODs (fruit fly, horse, house mouse, and human; see Table 1) using the NJ method of Molecular Evolutionary Genetics Analysis 4 (Figure 2; Tamura et al., 2007). The branch points represent speciation events designated $\mathrm{S}_{1}-\mathrm{S}_{3}$. Figure 2 shows that the 14 Fe-SODs (or Mn-SODs) from lower prokaryotes form a coherent grouping, whereas the $4 \mathrm{Cu} / \mathrm{Zn}$-SODs from eukaryotes form another grouping $\left(\mathrm{S}_{1}\right.$ separates eukaryotes from prokaryotes, implying the commencement of divergence between Fe-SOD/Mn-SOD and $\mathrm{Cu} / \mathrm{Zn}-\mathrm{SOD}$ within the common ancestry). In the prokaryote grouping, the SODs from Myxococcus xanthus DK 1622, Magnetospirillum magnetotacticum MS-1, and Acinetobacter sp ADP1 branch relatively early ( $\mathrm{S}_{2}$ splits algae from other prokaryote), but the analysis failed to distinguish an outgroup from Fe-SOD and Mn-SOD. In the eukaryote grouping, the SOD from the lower animal (fruit fly) branches relatively early $\left(\mathrm{S}_{3}\right)$, whereas those from mammals (horse, house mouse, and human) branch later. Together with the result from the immunological and phylogenetic analyses, these results suggest that the Fe-SODs (or Mn-SODs) and Cu/Zn-SODs are remote from each other evolutionarily and evolved in different directions. 
Table 1. Taxonomic placement and classes of superoxide dismutases (SODs) of each species and GenBank accession numbers included in the phylogenetic analyses.

\begin{tabular}{|c|c|c|c|c|}
\hline Codes & Group & Species & Classes of SODs & Accession No. \\
\hline 1 & Algae & Nostoc commune stain CHEN & Fe-SOD & AAV84021 \\
\hline 2 & Algae & Nostoc punctiforme PCC 73102 & $\mathrm{Fe}-\mathrm{SOD}$ & ZP_00108516 \\
\hline 3 & Algae & Nostoc commune stain DRH1 & Fe-SOD & $\mathrm{AAF} 25009$ \\
\hline 4 & Algae & Nostoc linckia & Fe-SOD & AAL25194 \\
\hline 5 & Algae & Nostoc sp PCC 7120 & Fe-SOD & NP 486978 \\
\hline 6 & Algae & Anabaena variabilis ATCC 29413 & Mn-SOD & ABA 20587 \\
\hline 7 & Algae & Plectonema boryanum UTEX 'B 485' & $\mathrm{Fe}-\mathrm{SOD}$ & AAA69954 \\
\hline 8 & Algae & Thermosynechococcus elongatus BP-1 & Mn-SOD & BAC09071 \\
\hline 9 & Algae & Synechococcus sp JA-3-3Ab & Fe-SOD & ABD00958 \\
\hline 10 & Algae & Synechococcus sp JA-2-3B'a(2-13) & $\mathrm{Fe}-\mathrm{SOD}$ & ABD03447 \\
\hline 11 & Prokaryote & Myxococcus xanthus DK 1622 & Mn-SOD & ABF87575 \\
\hline 12 & Prokaryote & Magnetospirillum magnetotacticum MS-1 & Mn-SOD & ZP_00054068 \\
\hline 13 & Prokaryote & Acinetobacter $\mathrm{sp}$ ADP1 & Fe-SOD & YP_0 046036 \\
\hline 14 & Algae & Spirulina platensis & $\mathrm{Fe}-\mathrm{SOD}$ & AAQ22734 \\
\hline 15 & Eukaryote & Drosophila melanogaster (fruit fly) & $\mathrm{Cu} / \mathrm{Zn}-\mathrm{SOD}$ & NP 476735 \\
\hline 16 & Eukaryote & Homo sapiens (human) & $\mathrm{Cu} / \mathrm{Zn}-\mathrm{SOD}$ & NP_000445 \\
\hline 17 & Eukaryote & Equus caballus (horse) & $\mathrm{Cu} / \mathrm{Zn}-\mathrm{SOD}$ & BAA76921 \\
\hline 18 & Eukaryote & Mus musculus (housemouse) & $\mathrm{Cu} / \mathrm{Zn}-\mathrm{SOD}$ & NP_035564 \\
\hline
\end{tabular}

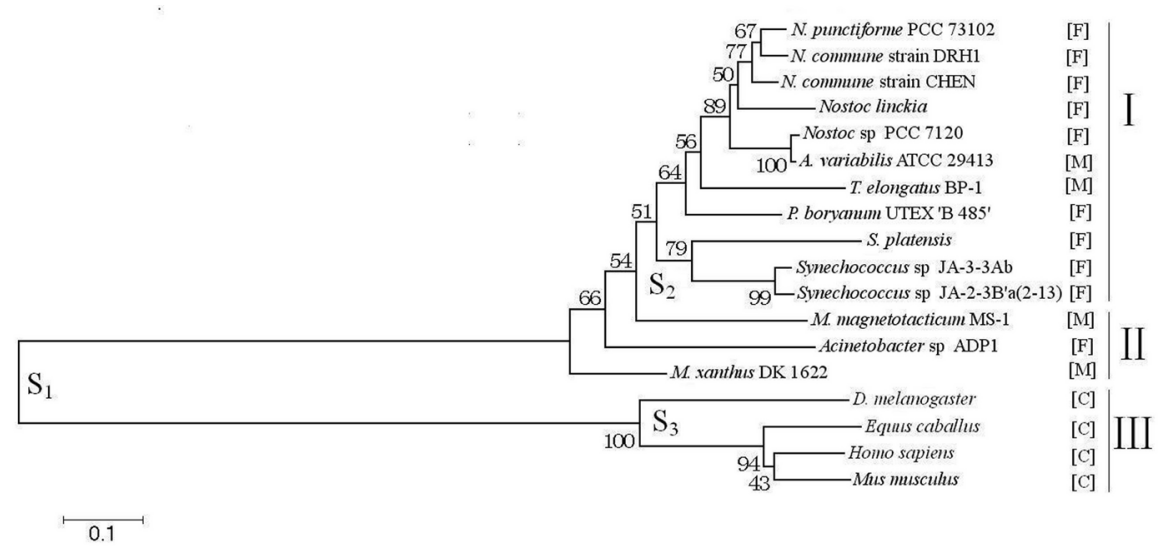

Figure 2. Phylogenetic relationships among members of the superoxide dismutase (SOD) family. Phylogenetic trees were constructed using the neighbor-joining method. The lengths of the branches reflect evolutionary distance. Number at the branch nodes indicates percentage bootstrap support for 3000 replicates. Speciation events are designated $\mathrm{S}_{1}, \mathrm{~S}_{2}$ and $\mathrm{S}_{3}$. The groups 'Algae', 'Prokaryote (without algae)', and 'Eukaryote' are shown by I, II and III, respectively. [F], [M] and [C] represent Fe-SOD, Mn-SOD and $\mathrm{Cu} / \mathrm{Zn}-\mathrm{SOD}$, respectively.

\section{Evolutive characterization of $N$. commune stain CHEN Fe-SOD}

Fourteen prokaryote SODs were aligned and compared with one another using the GeneDoc software (significant similarity was found with the $4 \mathrm{Cu} / \mathrm{Zn}$-SODs describe above; data not shown). As shown in Figure 3, of 200 common residues in the 14 SODs, 31 are variable, 121 are conserved, and 60 are absolutely conserved. In addition, 37 of the 60 absolutely conserved residues are from 3 conserved regions: GIFNNAAQVWNHTFFW (69-84), AAATQFGSGWAWL (120-132), and DVWEHAYY (162-169). According to the DVWEHAYY-conserved region, a downstream primer 
(5'-AATGTAGTAGGCGTCTTC-3') was designed, and we successfully amplified partial S. platensis fe-sod (accession No. AAQ22734) using genomic DNA (the upstream primer was above SODforward). In addition, we found 3 peculiar mutant residues (Ser81, Met162, and Asp166; see Figure 3 ) in the SOD from S. platensis, and these residues are absolutely conserved in the other 10 algal SODs. This heterogeneity introduces difficulty to the forecasting and designing of a downstream primer to clone integrated S. platensis fe-sod CDS (studies are proceeding now).
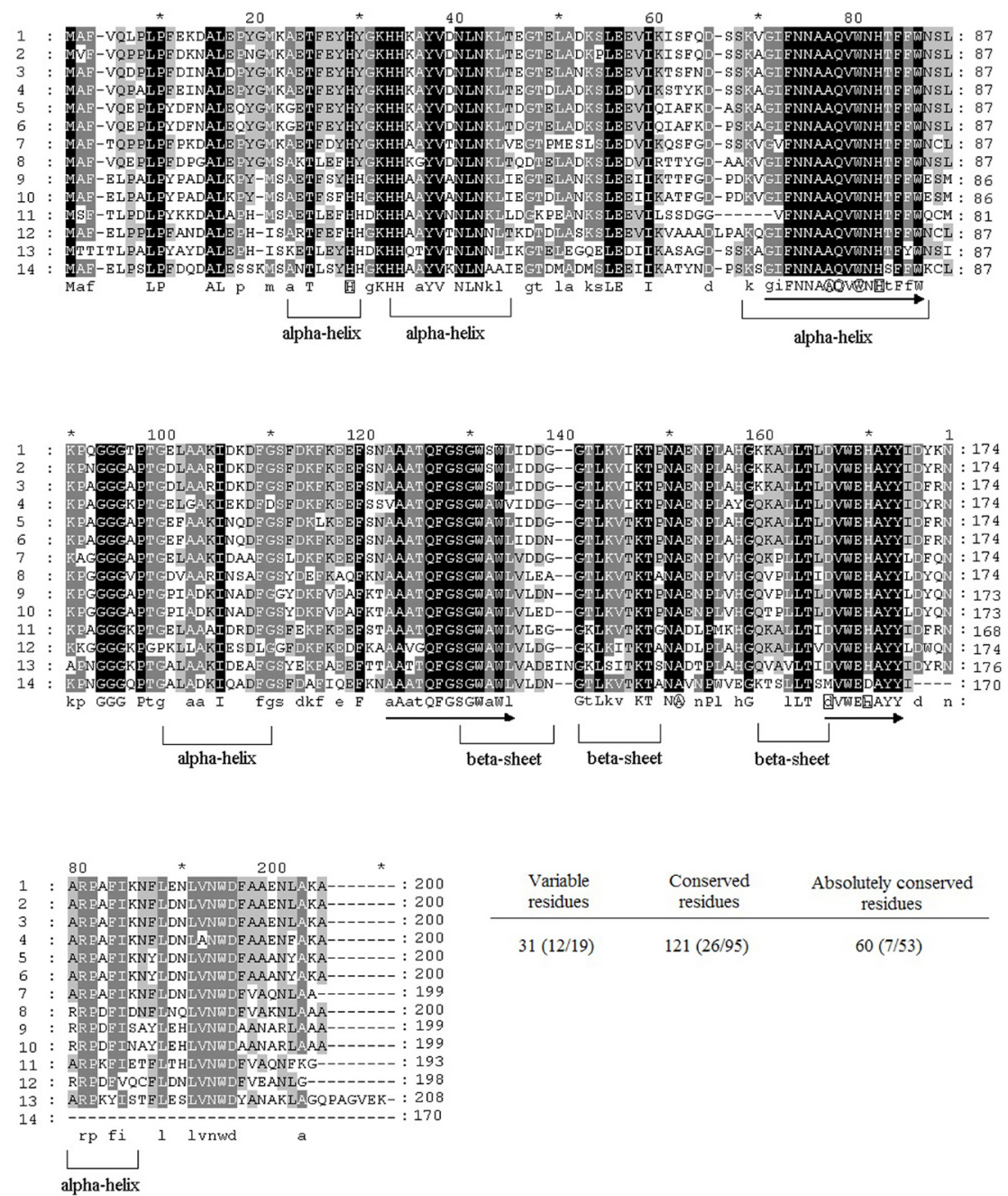

Figure 3. Alignment of superoxide dismutases (SODs) from 14 reported low prokaryote in Genbank. Variable residues are displayed as black letters on a white background; conserved residues are displayed as white letters on a gray grayground (subscript lowercase); absolutely conserved residues are displayed as white letters on a black background (subscript uppercase); three conserved regions are displayed as subscript arrowhead. The $\mathrm{Fe}^{3+}$ bound sites are in subscript box; characterized residues distinguishing Fe-SODs from Mn-SODs are in subscript circle. The followed entry in the matrix was concluded form alignment, indicating the total number of variable residues, conserved residues, and absolutely conserved residues from total 200 residues. The number in bracket indicates the number of corresponding residues in interior region (31-169 amino acids)/exterior region. 
Many important signature amino acid residues were also revealed: 2 unique and conserved amino acid residues (Thr138 and Asn149) distinguish algae from other lower prokaryotes (see Figure 2, $\mathrm{S}_{2}$ ); in addition, $N$. commune stain CHEN Fe-SOD exhibits 4 residues (Leu6, Gln90, Thr94, and Lys173) that are absent in other SODs. Further, the residues implicated in the formation of the active site and metal ion binding are absolutely conserved in these SODs - such as $4 \mathrm{Fe}^{3+}$-bound sites in the SOD active center (His28, His80, Asp162, and His 166; a water molecule or a hydroxyl ion is the fifth site) as well as the residues Ala75, Gln76, Trp78, and Ala147, which are proposed as primary candidates to distinguish Fe-SODs from Mn-SODs (Parker and Blake, 1988a,b; Van Camp et al., 1990). Interestingly, of 139 interior residues (we define 31-169 amino acids as the interior region and the others as the exterior region), 19 are variable, 95 are conserved, and 53 are absolutely conserved; each of the 3 conserved regions described above are included. However, of 61 exterior residues, 12 are variable, 26 are conserved, and only 7 are absolutely conserved. Conclusively, it is significant that the interior residues show a lower mutation rate than that of the exterior residues.

\section{Structure and function analysis}

The N. commune stain CHEN amino acid sequence was submitted to the SWISSMODEL server using ExPASy (Schwede et al., 2003; Kopp and Schwede, 2006), and the structure was predicted. Figure 4 shows that the structure can be divided into 2 domains, the N-terminal domain involving an extended region followed by 2 alpha-helices (Ala22Tyr29, His32-Tyr44) and the C-terminal domain containing 3 alpha-helices (Ser66-Asn79, Gly97-Gly108, and Arg176-Asn185), and 3 beta-sheets (Ser127-Gly136, Gly137-Pro145, and Lys155-Asp162) inserted sequentially between the fourth and fifth helices (see Figure 3). This structure is comparable to that in a report of an X-ray analysis of Fe-SOD from Mycobacterium tuberculosis, implying that the prediction is considerably reliable (Cooper et al., 1995).

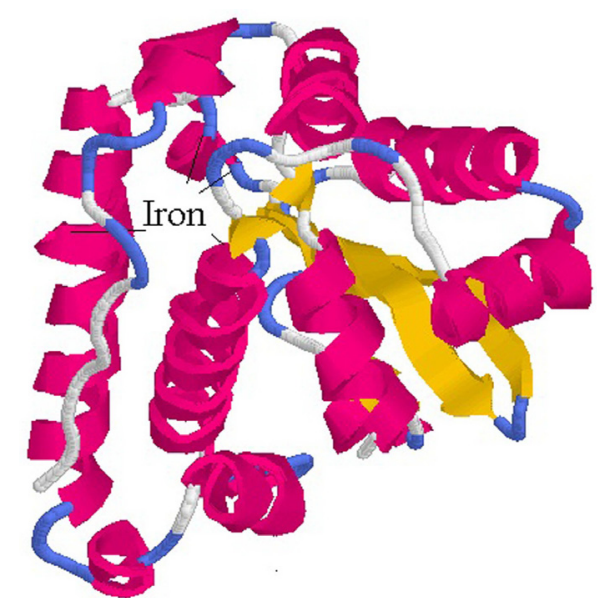

Figure 4. The tertiary structure of Fe-SOD from Nostoc commune stain CHEN by RasMol 2.6. The amino acid sequence of superoxide dismutase (SOD) was submitted to SWISS-MODEL server, so that the tertiary structure was predicted (BLAST search P $<0.00001$, SIM $>25 \%$ ). Alpha-helices, beta-sheet, and two ends of protein sequence are in pink, yellow, and blue, respectively. 
In addition, the $4 \mathrm{Fe}^{3}$-bound sites are close to one another even though they are apart in the primary structure. Further, the active center and $\mathrm{Fe}^{3+}$-bound sites are inside the SOD molecule, whereas the ends of the sequences (beside 31-169 amino acids) are outside and relatively loose. Accordingly, these ends also have a mutation rate higher than that of the interior (see Figure 3, matrix). To characterize this coincidence in detail, we compared N. commune stain CHEN Fe-SOD with 10 SODs from other algae and determined the mutation rates of various regions. Table 2 shows that the mutation rates of the exterior region in the 10 controls are significantly higher than those of the interior region on the protein level but relatively equal on the gene level.

\begin{tabular}{|c|c|c|c|}
\hline \multirow[t]{2}{*}{9 algae species ${ }^{\mathrm{a}}$} & Protein mutation rate $(\%)^{\mathrm{b}}$ & Gene mutation rate $(\%)^{\mathrm{b}}$ & Sequence identity (\%) \\
\hline & Exterior/interior ${ }^{\mathrm{c}}$ & Exterior/interior & Gene/protein \\
\hline 2 & $11.5 / 4.3$ & $8.6 / 6.2$ & $93 / 93$ \\
\hline 3 & $13.1 / 5.7$ & $7.7 / 9.8$ & $90 / 92$ \\
\hline 4 & $13.2 / 12.9$ & 13.1/13.7 & $87 / 87$ \\
\hline 5 & $18.0 / 11.5$ & 19.3/17.1 & $82 / 86$ \\
\hline 6 & $21.3 / 10.7$ & $21.1 / 15.8$ & $82 / 86$ \\
\hline 7 & $18.0 / 17.9$ & $22.4 / 25.7$ & $75 / 82$ \\
\hline 8 & $29.5 / 23.7$ & $29.5 / 30.7$ & $69 / 73$ \\
\hline 9 & $41.0 / 23.7$ & $34.9 / 28.4$ & $68 / 70$ \\
\hline 10 & $41.0 / 24.5$ & $34.0 / 30.5$ & $67 / 69$ \\
\hline
\end{tabular}

${ }^{\mathrm{a}}$ The codes of the 9 algae species are the same with Table $1 .{ }^{\mathrm{b}}$ Mutation rate $=$ number of variable residues (or nucleotides) / number of total residues (or nucleotides) x 100\%. ${ }^{\circ}$ Exterior: region beside of 31-169 amino acids (or 93-507 nucleotides). Interior: region of 31-169 amino acids (or 93-507 nucleotides).

\section{DISCUSSION}

When gene sequences initially begin to diverge during evolution, mutations usually accumulate most rapidly in the third codon, and most substitutions are synonymous. As a result, nucleotide identity between 2 gene sequences is usually lower than amino acid identity between the 2 corresponding proteins. Typical of this situation are the $p$-vac and $c$-vac genes of Halobacterium halobium and the c-vac gene of Haloferax mediterranei (Horne et al., 1988; Englert et al., 1990). For these genes, the gene identity is much lower than the corresponding amino acid identity ( 84.7 vs $97.4 \%, 86.4$ vs $96.1 \%$, and 85.5 vs $98.7 \%$, respectively). However, the algal sods do not conform to behavior displayed in these genes. The Basic Local Alignment Search Tool 2 sequence results of the comparison of $N$. commune CHEN Fe-SOD with 10 other algal SODs, sods showed relatively equivalent gene and amino acid identities $(67-93 \%$ identity between gene sequences and 69-93\% identity between amino acid sequences; see Table 2). These results imply that most of the encoding triplets are connected by nonsynonymous nucleotide substitutions, which indicates that these sods from algae continue to be subjected to strong and changing selection. This selection may be related to intracellular or extracellular stress from oxygen radicals.

Interestingly, this strong selection seems to lead to a special evolutive result: the conserved residues are mostly localized on the interior of protein sequences, whereas the exterior parts exhibit a higher degree of variability. A similar phenomenon was reported in 8 sets of homol- 
ogous protein families as early as 1980 (Go and Miyazawa, 1980), but the mechanism remains unclear. The statistics in Table 2 present this evolutive characterization in detail. We designed an $\mathrm{Re} / \mathrm{Ri}$ (mutation rate of exterior/mutation rate of interior) for the data in Table 2 to visualize the diversity of mutation rates in various regions. An $\mathrm{Re} / \mathrm{Ri}$ of $>1$ represents an exterior mutation rate that is higher than that of the interior, whereas an Re/Ri of 1 implies random mutation. The $\mathrm{Re} / \mathrm{Ri}$ fluctuated near 1 on the gene level, implying that nucleotide mutations occur randomly on the entire sequence; however, the $\mathrm{Re} / \mathrm{Ri}$ of the 10 proteins studied were all above 1 to varying degrees. Remarkably, the $\mathrm{Re} / \mathrm{Ri}$ is high in 2, 3, and 6 controls, suggesting a significant imbalance of mutation distribution in these species. Given the distinct $\mathrm{Re} / \mathrm{Ri}$ values on the gene and protein levels, we can easily conclude that of the total equally distributed nucleotide substitutions on the gene level, most of the nonsynonymous substitutions accumulate at the ends of gene sequences, leading to higher mutation rate on the exterior of the protein, whereas synonymous substitutions are mostly localized at the interior of the gene, inducing a conserved protein interior.

We attribute this imbalanced distribution of nonsynonymous mutation to a special law of nature. According to this law, exterior-mutating proteins tend to survive, whereas interiormutating proteins are likely to be eliminated. We tried to uncover the special law of nature using associative analysis of its structure and function: nonsynonymous substitutions localized at the gene interior usually destroy the enzyme active center. Figures 3 and 4 show the sites of catalysis, and $\mathrm{Fe}^{3+}$-bound sites are all between the 31-169-amino acid region, constituting the enzyme activity center; they are absolutely conserved. Subsequently its antioxidant function is impaired. These substitutions go against the special law of nature and are therefore likely to be eliminated. On the contrary, although exterior nonsynonymous substitutions on sod also lead to exteriormutating proteins, they do not impair enzymatic activity because of their special structure. Figure 4 shows that the exterior residues are on the surface of the protein and well removed from the site of catalysis; they are not presumed to participate in the formation of the enzyme activity center. Hence, these substitutions survive under the special law of nature. In a word, this law was function-based during algal SOD evolution. We attribute the action of the special law of nature to an attempt by the organism to maintain enzymatic catalytic function and survive oxidative stress. Accordingly, we fused an $S c F v$ gene fragment on one end of $N$. commune fe-sod, which was presumed to be enzyme function-independent, and the resulting fusion protein retained considerable enzyme activity but had higher anti-tumor efficacy (Lu et al., 2006).

In sum, the fe-sod CDS from $N$. commune stain CHEN was cloned, and its gene and protein sequences exhibited many unusual and remarkable features: 1) the rare nonsynonymous substitutions within algal sods implied that they are being subjected to strong natural selection; 2) under this strong selection, although the mutation was random on the gene level, on the protein level, the exterior region is inclined to mutate $(\mathrm{Re} / \mathrm{Ri}>1)$ owing to the higher frequency of nonsynonymous substitutions in the exterior region; and 3) the interesting evolution characterization was function-based to maintain SOD activity under strong natural selection. These features show the theoretical feasibility of modifying the ends of Fe-SOD to overcome its disadvantages in clinical applications.

\section{ACKNOWLEDGMENTS}

We thank Dr. Jiong Chen for a critical reading of the manuscript. Research supported by a grant from the National Natural Science Foundation of China (\#30900760). 


\section{REFERENCES}

Behrend L, Henderson G and Zwacka RM (2003). Reactive oxygen species in oncogenic transformation. Biochem. Soc. Trans. 31: 1441-1444.

Brioukhanov AL, Nesatyy VJ and Netrusov AI (2006). Purification and characterization of Fe-containing superoxide dismutase from Methanobrevibacter arboriphilus strain AZ. Biochemistry 71: 441-447.

Chen X, Kodama T, Iida T and Honda T (2007). Demonstration and characterization of manganese superoxide dismutase of Providencia alcalifaciens. Microbiol. Immunol. 51: 951-961.

Choudhary M, Jetley UK, Abash KM, Zutshi S, et al. (2007). Effect of heavy metal stress on proline, malondialdehyde, and superoxide dismutase activity in the cyanobacterium Spirulina platensis-S5. Ecotoxicol. Environ. Saf. 66: 204-209.

Cooper JB, McIntyre K, Badasso MO, Wood SP, et al. (1995). X-ray structure analysis of the iron-dependent superoxide dismutase from Mycobacterium tuberculosis at 2.0 Angstroms resolution reveals novel dimer-dimer interactions. $J$. Mol. Biol. 246: 531-544.

Delanian S, Martin M, Bravard A, Luccioni C, et al. (2001). Cu/Zn superoxide dismutase modulates phenotypic changes in cultured fibroblasts from human skin with chronic radiotherapy damage. Radiother. Oncol. 58: 325-331.

Englert C, Horne M and Pfeifer F (1990). Expression of the major gas vesicle protein gene in the halophilic archaebacterium Haloferax mediterranei is modulated by salt. Mol. Gen. Genet. 222: 225-232.

Go M and Miyazawa S (1980). Relationship between mutability, polarity and exteriority of amino acid residues in protein evolution. Int. J. Pept. Protein Res. 15: 211-224.

Gottlieb MG, Schwanke CH, Santos AF, Jobim PF, et al. (2005). Association among oxidized LDL levels, MnSOD, apolipoprotein E polymorphisms, and cardiovascular risk factors in a south Brazilian region population. Genet. Mol. Res. 4: 691-703.

Horne M, Englert C and Pfeifer F (1988). Two genes encoding gas vacuole proteins in Halobacterium halobium. Mol. Gen. Genet. 213: 459-464.

Hu Y, Rosen DG, Zhou Y, Feng L, et al. (2005). Mitochondrial manganese-superoxide dismutase expression in ovarian cancer: role in cell proliferation and response to oxidative stress. J. Biol. Chem. 280: 39485-39492.

Jubeh TT, Antler S, Haupt S, Barenholz Y, et al. (2005). Local prevention of oxidative stress in the intestinal epithelium of the rat by adhesive liposomes of superoxide dismutase and tempamine. Mol. Pharm. 2: 2-11.

Kim CS, Lee CH, Shin JS, Chung YS, et al. (1997). A simple and rapid method for isolation of high quality genomic DNA from fruit trees and conifers using PVP. Nucleic Acids Res. 25: 1085-1086.

Kopp J and Schwede T (2006). The SWISS-MODEL Repository: new features and functionalities. Nucleic Acids Res. 34 : D315-D318.

Li J, Zhou K, Meng X, Wu Q, et al. (2008). Increased ROS generation and SOD activity in heteroplasmic tissues of transmitochondrial mice with A3243G mitochondrial DNA mutation. Genet. Mol. Res. 7: 1054-1062.

Lu M, Gong X, Lu Y, Guo J, et al. (2006). Molecular cloning and functional characterization of a cell-permeable superoxide dismutase targeted to lung adenocarcinoma cells. Inhibition cell proliferation through the Akt/p27kip1 pathway. J. Biol. Chem. 281: 13620-13627.

Marklund S and Marklund G (1974). Involvement of the superoxide anion radical in the autoxidation of pyrogallol and a convenient assay for superoxide dismutase. Eur. J. Biochem. 47: 469-474.

Oyanagui Y, Sato S and Inoue M (1991). Inhibition of carrageenan-induced paw edema by superoxide dismutase that binds to heparan sulfates on vascular endothelial cells. Biochem. Pharmacol. 42: 991-995.

Parker MW and Blake CC (1988a). Crystal structure of manganese superoxide dismutase from Bacillus stearothermophilus at 2.4 A resolution. J. Mol. Biol. 199: 649-661.

Parker MW and Blake CC (1988b). Iron- and manganese-containing superoxide dismutases can be distinguished by analysis of their primary structures. FEBS Lett. 229: 377-382.

Regelsberger G, Laaha U, Dietmann D, Ruker F, et al. (2004). The iron superoxide dismutase from the filamentous cyanobacterium Nostoc PCC 7120. Localization, overexpression, and biochemical characterization. J. Biol. Chem. 279: 44384-44393.

Rengel RG, Filipovic-Grcic J, Cepelak I, Zanic-Grubisic T, et al. (2005). The effect of liposomes with superoxide dismutase on A2182 cells. Eur. J. Pharm. Biopharm. 60: 47-51.

Schwede T, Kopp J, Guex N and Peitsch MC (2003). SWISS-MODEL: An automated protein homology-modeling server. Nucleic Acids Res. 31: 3381-3385.

Seo SN, Lee JH and Kim YM (2007). Characterization of an iron- and manganese-containing superoxide dismutase from Methylobacillus sp strain SK1 DSM 8269. Mol. Cells 23: 370-378.

Shirkey B, Kovarcik DP, Wright DJ, Wilmoth G, et al. (2000). Active Fe-containing superoxide dismutase and abundant

Genetics and Molecular Research 11 (4): 3607-3617 (2012)

CFUNPEC-RP www.funpecrp.com.br 
sodF mRNA in Nostoc commune (Cyanobacteria) after years of desiccation. J. Bacteriol. 182: 189-197.

Takenaka S, Koshiya J, Okugawa S, Takata A, et al. (2011). Fe-superoxide dismutase and 2-hydroxy-1,4-benzoquinone reductase preclude the auto-oxidation step in 4-aminophenol metabolism by Burkholderia sp strain AK-5. Biodegradation 22: 1-11.

Tamura K, Dudley J, Nei M and Kumar S (2007). MEGA4: Molecular Evolutionary Genetics Analysis (MEGA) software version 4.0. Mol. Biol. Evol. 24: 1596-1599.

Van CW, Bowler C, Villarroel R, Tsang EW, et al. (1990). Characterization of iron superoxide dismutase cDNAs from plants obtained by genetic complementation in Escherichia coli. Proc. Natl. Acad. Sci. U. S. A. 87: 9903-9907.

Zhang Y, Zhao W, Zhang HJ, Domann FE, et al. (2002). Overexpression of copper zinc superoxide dismutase suppresses human glioma cell growth. Cancer Res. 62: 1205-1212. 